livraisons

d'Histoire

de l'Architecture

\section{Livraisons de l'histoire de l'architecture}

$37 \mid 2019$

L'architecture mise à l'examen

\title{
Les concours d'architecture en URSS (1917-1953) : entre innovations et régressions
}

Architectural competitions in the USSR (1917-1953): between innovations and regressions

Die Architekturwettbewerbe in der Sowjetunion (1917-1953): zwischen

Innovation und Rückschritt

\section{Stéphane Gaessler}

\section{OpenEdition}

Journals

Édition électronique

URL : http://journals.openedition.org//ha/1402

DOI : $10.4000 /$ /ha. 1402

ISSN : 1960-5994

\section{Éditeur}

Association Livraisons d'histoire de l'architecture - LHA

Édition imprimée

Date de publication : 15 juin 2019

Pagination : 85-95

ISSN : 1627-4970

Référence électronique

Stéphane Gaessler, «Les concours d'architecture en URSS (1917-1953) : entre innovations et régressions », Livraisons de l'histoire de l'architecture [En ligne], 37 | 2019, mis en ligne le 02 janvier 2021, consulté le 26 janvier 2021. URL : http://journals.openedition.org/lha/1402 ; DOI : https:// doi.org/10.4000/lha.1402 


\section{LES CONCOURS D'ARCHITECTURE EN URSS (1917-1953) : ENTRE INNOVATIONS ET RÉGRESSIONS}

\section{Les concours, creusets d'une architecture soviétique?}

La pratique des concours d'architecture remonte en Russie au XVIII ${ }^{e}$ siècle ${ }^{1}$, avant de se généraliser à partir de la deuxième moitié du XIX ${ }^{e}$ siècle. On observe à cette période, une diversification des commanditaires et des organisateurs, qui ne sont plus uniquement le tsar ou l'Académie des Beaux-Arts, mais aussi des autorités locales, par exemple, la Douma municipale de Moscou (concours du Musée Historique en 1874 et des galeries commerçantes de la place rouge en 1888), et principalement des commanditaires privés. On voit apparaitre des associations professionnelles indépendantes, comme la MAO (Société architecturale de Moscou), créée en 1867, qui organise ses premiers concours dès 1868. Les années qui précèdent la Première guerre mondiale, connaissent un véritable boum de la construction, qui conduit à l'organisation d'un nombre significatif de concours d'architecture, 177 entre 1911 et $1914^{2}$. Après la révolution et la guerre civile, le passage de la politique du " communisme de guerre » à celle de la $\mathrm{NEP}^{3}$, introduit une libéralisation partielle de la société qui renoue avec la croissance économique. Les chantiers sont relancés, ainsi que les discussions théoriques et les recherches expérimentales sur l'architecture et l'urbanisme, dans une Russie en pleine transformation. Ces deux moments se retrouvent dans la pratique des concours d'architecture. Le concours devient une étape incontournable du processus de mise en œuvre des projets d'architecture et d'urbanisme en URSS. Igor Kazus' en recense un total de 955 entre 1917 et $1941^{4}$. Moments de compétition certes, mais aussi interfaces de débat collectif sur les problématiques architecturales et urbanistiques, dont les résultats sont publiés et débattus dans la presse, ils seront les lieux privilégiés d'expression et d'affirmation des courants d'avant-garde.

Les concours servent d'abord à définir de nouvelles typologies architecturales, éléments constitutifs d'une transformation socialiste de la société russe. C'est le cas, par exemple, de la typologie de la Maison du peuple (concours pour la Maison du peuple d'Ivanovo-Voznesensk en 1924), ou de celle de la Maison des soviets, siège

1. Le premier concours d'architecture organisé en Russie serait celui pour le bâtiment des douze collèges, à Saint-Pétersbourg, en 1723-1724.

2. I. G. Kazus', Sovietskaja arhitektura 1920x godov: organizacija proektirovanja [L'architecture soviétique des années 1920 : organisation de la conception], Moscou : Directmedia, 2013, p. 79.

3. Nouvelle politique économique à partir de mars 1921.

4. I. G. Kazus', op. cit., p. 98. 


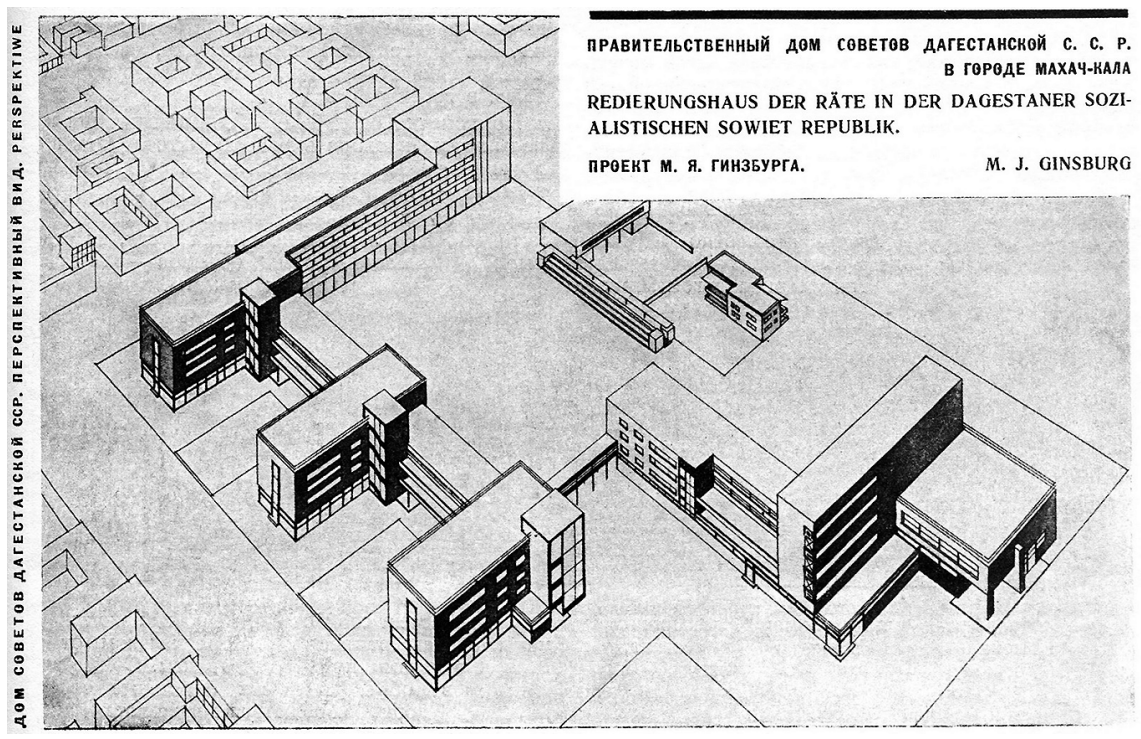

Ill. 1 : Moïsseï Ginzburg (1892-1946), Projet de concours pour la Maison des soviets de la République du Dagestan à Makhatchkala, 1926, Sovremmenaja arhitektura, 1926, nº 5-6, p. 113-115. () D.R.

des organes du pouvoir local soviétique (concours de 1924 pour la Maison des soviets de Briansk). Une série de concours pour des "maisons de gouvernement", sièges de l'administration des différentes républiques de l'URSS, inaugurent notamment une réflexion sur ce que doit être une architecture soviétique dans les territoires non russes, comme lors du concours de la Maison des soviets de la république du Dagestan, en 1926, qui confrontera Ivan Joltovski (1867-1959) et Moïsseï Ginzburg (1892-1946) (ill. 1). À côté des architectures ayant une fonction essentiellement représentative et symbolique, les concours porteront principalement sur des constructions utilitaires, des complexes de logements, des infrastructures socioculturelles (telles qu'écoles, crèches, cuisine-fabriques, clubs ouvriers, cinémas, théâtres, palais de la culture, mais aussi centres commerciaux et banques), des institutions d'enseignement et de formation, des hôpitaux, des maisons coopératives, des crématoriums, des bains publics, des espaces verts et de divertissement comme la typologie des parcs de culture et de repos.

Les concours d'architecture permettent de suivre l'évolution du thème du logement en URSS. Dès 1917, plusieurs concours portent sur la création d'ensembles résidentiels, entre autres pour les employés des chemins de fer, où prédominent les exemples de cités-jardins. En septembre 1922, la municipalité de Moscou commande à la $\mathrm{MAO}$, un concours pour la conception de deux ensembles de logements ouvriers "modèles" (Pokazatel'nye), ayant la particularité de prévoir des immeubles séparés pour les familles et les célibataires. Ce concours fera date avec la participation d'architectes comme Konstantin Melnikov (1890-1974), les frères Panteleïmon et Ilya Golossov ou encore Andreï Belogrud (1875-1933). Il s'agit de développer un 


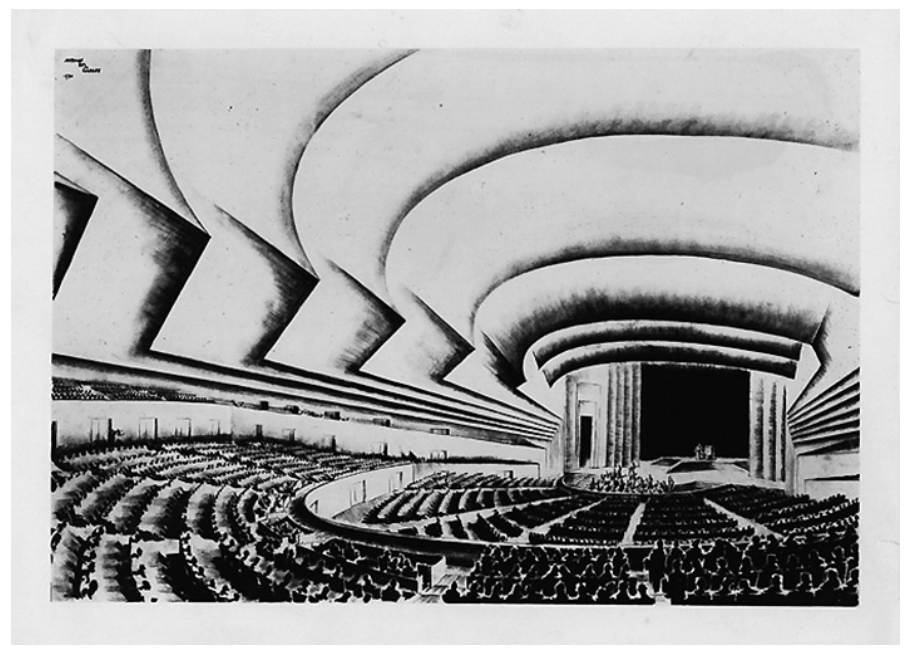

Ill. 2 : Norman Bel Geddes (1893-1958), Projet de concours pour un théâtre musical de masse à Kharkov, auditorium de 4000 places, 1930-1932, deuxième prix. (C) Collections du Harry Ransom center, Université d'Austin au Texas.

ensemble résidentiel, dans le cadre des premières opérations de restructurations urbaines du Plan Novaja Moskva (Nouveau Moscou, 1918-1923), mais aussi d'expérimenter une forme d'habitat social, qui reste encore très proche des modèles développés en Europe à la même époque. En 1925-1926, la municipalité de Moscou organise deux concours sur des projets de logements ouvriers, introduisant une interprétation socialiste beaucoup plus radicale de l'habitat. Le deuxième concours, remporté par l'architecte Vladimir Majat (1876-1954), introduit la typologie de la Maison-Commune, pensée comme un condensateur social impliquant un haut degré de collectivisation des espaces, et l'aménagement de nombreux services aux résidents.

Les concours d'architecture se situent au croisement d'un certain nombre de fonctions utilitaires, expérimentales, d'apprentissage (concours internes aux VKUTEMAS 5 ) et de prestige, débouchant notamment sur une première internationalisation de l'architecture soviétique. En 1930-1931, le concours pour un théâtre musical de 4000 places à Kharkov (ill. 2), reçoit près d'une centaine de projets étrangers, et attire des noms illustres, comme Walter Gropius (1883-1969), les Américains Alfred Kastner (1901-1975), Oscar Storonov (1905-1970), Norman Bel Geddes (1893-1958), ou encore le Japonais Ranahichiro Kawakita (1902-1975). Les concours ne donneront pas forcément lieu à réalisation, mais auront souvent une incidence considérable sur le développement théorique et sur la documentation urbanistique. En 1922-1923, le concours pour le Palais du travail, pensé comme un nouveau centre civique de la capitale russe, ne sera jamais construit, mais il aura un retentissement très important, en faisant connaître au monde l'architecture constructiviste. Si le

\section{Ateliers supérieurs d'art de technique.}


concours de 1922 portant sur le plan d'aménagement de l'exposition agricole et artisanale panrusse de Moscou, a pour objet la création d'un ensemble éphémère, il constitue aussi la première étape de l'aménagement du futur Parc central de culture et de repos Maxime Gorki. Bien plus encore, le concours pour le Palais des soviets (1931-1933), déterminera pour plusieurs décennies, les grandes lignes de développement du centre de Moscou, sans qu'il ne soit jamais lui-même réalisé.

La thématique urbaine culmine dans la série de concours organisés pour des villes nouvelles, au moment de l'intense débat professionnel sur la répartition territoriale socialiste de la population (Socrasselenie), en 1929-1930, qui oppose principalement l'approche "désurbaniste », théorisée par Mikhail Okhitovič (1896-1937), et l'approche " urbaniste ", théorisée par Leonid Sabsovič. En 1930, le concours portant sur la "ville verte" et celui pour la ville nouvelle de Magnitogorsk, vont en particulier permettre de confronter ces deux approches théoriques, avec pour résultat la censure officielle du désurbanisme. Mikhail Okhitovič sera assassiné par le NKVD en 1937.

En 1932, un concours d'idées est lancé comme première étape de l'élaboration du futur plan général de Moscou ${ }^{6}$. Plusieurs architectes étrangers y participent : Le Corbusier, Ernst May (1886-1970) et Hannes Meyer (1889-1954). Aucun des plans proposés par ces représentants du mouvement moderne, ne seront approuvés. Les autorités préféreront confier la conception d'un plan-esquisse à l'architecte en chef de Moscou, Vladimir Nikolaevič Semenov (1874-1960), qui produit une synthèse intéressante de l'expérience haussmannienne et des idées de la cité-jardin. Ce planesquisse est la première formulation des principes directeurs de la doctrine urbanistique stalinienne, incarnée dans le Plan général de reconstruction de Moscou, adopté en juillet 1935. Avec l'évolution technologique de la construction, les concours acquièrent une valeur nouvelle, en devenant une étape indispensable dans la conception et l'adoption de projets-types destinés à la sérialisation. L'un des premiers concours de ce type est organisé en 1927 par la MAO pour l'élaboration de projetstypes pour des clubs de cheminots, à une époque où la standardisation est encore très partielle. Avec le lancement des premiers plans quinquennaux, on assiste à une accélération de l'industrialisation des processus de construction. Cette catégorie de concours deviendra de plus en plus prépondérante dans l'après-guerre.

\section{L'évolution d'une pratique professionnelle et de son institutionnalisation}

Un début de normalisation de la pratique des concours, au XIX ${ }^{\mathrm{e}}$ siècle et au début du $\mathrm{XX}^{\mathrm{e}}$ siècle, avait produit des protocoles de fonctionnement, encore mal définis et loin d'être universellement admis. Après la révolution, les organisations professionnelles, et notamment les représentants des mouvements d'avant-garde, vont s'attacher à donner de nouvelles règles et de nouveaux objectifs aux concours

6. A. Zaslavskij et A. Kozelkov. "Planirovka Moskvy» [Planification de Moscou] //Stroitel'stvo Moskvy, 1932, no 10, p. 6-19. 
d'architecture. Dès juillet 1918, l'architecte Iakov Reich (1883-1957) propose, dans un exposé au Syndicat des architectes de Moscou, de créer une commission pour élaborer une nouvelle règlementation des concours d'architecture, en s'appuyant sur l'expérience des règlements établis antérieurement en Russie et à l'étranger ${ }^{7}$. Survivante de la révolution, la Société architecturale moscovite (MAO) organise pas moins de 45 concours entre 1922 et 1930, contribuant à l'actualisation de cette pratique. Le premier concours organisé après la révolution par la MAO, celui du Palais du travail (1922-1923) fut décisif pour départager les deux grandes tendances architecturales qui coexistent et s'affrontent à cette période : le courant " rétrospectiviste", se situant dans la continuité de l'école néo-classique des années 1910, et l'avant-garde, incarnée par les groupes constructiviste et rationaliste. L'enjeu était de montrer que l'URSS suivait un chemin radicalement moderne en architecture. Malheureusement, les résultats du concours donnèrent l'impression tout à fait inverse, le premier prix étant attribué au projet historicisant de Noï Trotski (1895-1940), tandis que le projet constructiviste des frères Vesnin, salué par la nouvelle critique, reçut seulement le troisième prix. Le critique constructiviste Kornelii Zelinskij (1896-1970) écrivait "qu'il serait honteux de construire au centre de la Moscou rouge, capitale possible d'une Union mondiale de républiques soviétiques, un palais, qui par sa physionomie serait tourné vers le passé » ${ }^{8}$. En 1925, le concours pour le Télégraphe de Moscou, se soldait par l'attribution du chantier à un architecte, Ivan Rerberg (1869-1932), qui avait présenté un projet en dehors du concours. Dans son article "Comment on ne doit pas construire ", la revue constructiviste $S A$ (Architecture contemporaine), dénoncera le choix fait par le Ministère des Postes qui, n'ayant pas respecté les résultats du concours en faveur du projet constructiviste d'Aleksandr Grinberg, préféra l'architecture " rétrograde " de Rerberg9?

Les concours occupent à partir de ce moment une place centrale dans les débats d'architecture. Le 2 mai 1923, lors d'une assemblée générale de la MAO, Edgar Norvert (1884-1950) présente un exposé intitulé «À propos de l'organisation des concours d'architecture " ${ }^{10}$. La même année, la section des techniciens et ingénieurs du Syndicat des constructeurs, ouvrira une discussion sur les concours ${ }^{11}$. Ce qui ressort principalement de ces débats, c'est la volonté de donner au concours la valeur d'une procédure d'utilité publique, perdant une fonction essentiellement consultative, pour devenir pleinement un outil de sélection, expert et démocratique, de projets d'architecture voués à être réalisés. Les organisations professionnelles, comme

7. I. G. Kazus', op. cit., p. 70.

8. K. L. Zelinskij, "Stil' i stal' -k postrojke Dvorca SSSR v Moskve " [Le style et l'acier - à propos de la construction du Palais de l'URSS à Moscou] // Izvestia VCIK. $1^{\text {er }}$ juin 1923, cité dans Igor Kazus', op. cit., p. 120.

9. Rédaction de SA, "Kak ne nado stroit' " [Comment on ne doit pas construire]// SA, 1928, $\mathrm{n}^{\circ} 2$, p. 41-43.

10. P. V. Antipov, "O moskovskom arhitekturnom Obșestve » [Sur la Société architecturale moscovite] // Arhitektura, MAO, 1923, nº 3-5, p. 68-69.

11. A. P. Rojanovič , "K voprosu ob organizacii konkursnovo dela " [À propos de l'organisation des concours] // Stroitel’naja promyšlennost, 1926, nº 2, p. 139. 
la $\mathrm{MAO}$, y voient également un moyen d'assurer aux architectes une rémunération, dans des périodes de vaches maigres, et d'établir un mécanisme fiable de reconnaissance des droits d'auteur. Enfin le concours, instrument participatif par excellence, permet d'inscrire l'architecture dans le débat public, incitant les populations à s'intéresser et à prendre part aux processus de transformation de leur cadre de vie. L'architecte Boris Velikovski (1878-1937), propose par exemple aux Coopératives de logements, d'organiser des concours pour établir tous les nouveaux projets d'ensembles de logements ${ }^{12}$. En mai 1926, le premier Congrès pan-soviétique pour la construction technique et civile aborde la question de l'utilité méthodologique et pratique du concours. Deux points de vue s'y affrontent : celui étatiste, d'Aleksandr Rozenberg (1877-1935), du Syndicat des constructeurs, qui pense le concours, comme un instrument d'efficacité au service de l'État, recommandant la mise en place de jurys issus du STO (Conseil du travail et de la défense de l'URSS). Pour Rozenberg le concours doit permettre principalement de produire une idée-esquisse générale au premier stade de conception d'un projet ${ }^{13}$. Les associations professionnelles défendent une position différente, exprimée par l'architecte Andreï Ol' (1883-1958), de la Société des architectes de Léningrad: Ol' revendique une totale indépendance des concours vis-à-vis des structures étatiques. Il préconise que l'encadrement et la logistique soient assurés par des associations de professionnels, en s'appuyant sur une charte commune, et que le programme ne contienne aucune indication de préférence stylistique, se limitant aux valeurs économiques et fonctionnelles d'exploitation du projet. L'ASNOVA (Association des nouveaux architectes) proposa aussi de calquer les concours sur le modèle des procédures judiciaires (parties, avocats, juge) ${ }^{14}$. En 1926, lors d'une conférence donnée au siège de la MAO, l'architecte allemand Bruno Taut (1880-1938), introduit l'idée d'une évaluation horizontale par la "critique collective", brisant les rapports hiérarchiques entre concurrents, jury et public ${ }^{15}$. Finalement, en mai 1928, la MAO, adopte une nouvelle réglementation des concours, proposant un système de notation par points, et une plus grande transparence dans l'énonciation des principes qui régissent l'évaluation. Cette méthode est expérimentée lors du concours pour le centre commercial Mostorg à Moscou, en 1928, et reste en vigueur jusqu'au début des années $1930{ }^{16}$.

Les avancées obtenues en matière de transparence, de professionnalisme et d'expertise, sont vite recouvertes par le processus d'étatisation du système professionnel, qui est enclenché progressivement à partir de la deuxième moitié des années 1920.

12. B. Velikovskij, "Arhitekturnye konkursy v zhilstroitel'stve" [Les concours d'architecture pour la construction de logements] // Stroitel'naja promyšlennost', 1925, no 1, p. 3-4.

13. A. Rozenberg, "Sovremennaja praktika konkursnovo dela " [Pratique contemporaine des concours] // Stroitel'naja promyšlennost, 1926, nº 11, p. 788-791.

14. "V zasiitu konkurentov" // Ivestia ASNOVA, 1926, nº 1, p. 7.

15. O. Vutke, "Na doklade Bruno Taut v Moskovskom arhitekturnom Obšestve " [À propos de l'intervention de Bruno Taut à la MAO] // Stroitel'naja promyšlennost, 1926, nº 6-7, p. 466.

16. V. Serbakov, "Konkurs na zdanie univermaga Mostorga " [Concours pour le bâtiment de l'Univermag Mostorg] // Stroitel'stvo Moskvy, 1928, n 12, p. 3-7. 
L'unification des associations architecturales indépendantes au sein d'abord du VANO (Société scientifique d'architecture pan-soviétique) en 1930, puis de l'Union des architectes à partir de 1932, conjuguée à la création de grand trusts d'État et à la mainmise du NKVD sur une grande part de la planification urbanistique, amènent à privilégier les concours fermés et les concours commandés à des ateliers choisis (Zakaznye Konkursy), au détriment des concours ouverts. Les concours fermés et sur commande, s'accordent mieux avec l'établissement de relations plus hermétiques entre concepteurs et commanditaires de l'État. D'autre part, le processus de fonctionnarisation des architectes rendait caduc le principe même de la compétition ouverte. Les concours devaient se métamorphoser en instruments au service des besoins pratiques et des desseins idéologiques du pouvoir central.

Avec le concours pour le Palais des soviets, l'État prend un virage esthétique en faveur de "la réappropriation des formes du passé ». Dès le 28 février 1931, le jury recommande explicitement "l'utilisation des meilleures formes de l'architecture classique, tout en se fondant sur les avancées de la technique architecturale moderne ${ }^{17}$, avant d'attribuer les trois premiers prix aux projets art déco et classicisants d'Hector Hamilton, Boris Iofan et Ivan Joltovski. Le deuxième tour du concours du Palais des soviets expérimente une nouvelle méthode, qui deviendra l'une des caractéristiques des concours staliniens : fusionner les équipes ayant reçu les premiers prix, dans ce cas précis, Boris Iofan (1891-1976), Vladimir Ŝko (18781939) et Vladimir Gel'freih (1885-1967), afin de créer une synthèse des meilleures propositions. L'absence d'une définition claire de l'architecture dite " réaliste-socialiste ", conduit à transformer les concours en une méthode de conception architecturale cumulative qui a principalement pour objet de produire un répertoire d'idées où les architectes pourront ensuite puiser et développer leurs interprétations du passé et de la modernité. En 2014, l'exposition du Musée d'architecture de Moscou, Concours : forge de grande architecture, a bien montré cette fonction paradoxale des concours comme creusets de formes monumentale qui, tout en incarnant le système totalitaire, avaient pu être le réceptacle de l'imagination diverse des architectes. Les grands concours organisés à Moscou dans les années 1930 ont pour caractéristique de porter essentiellement sur des opérations d'embellissement de portions de ville (places et grandes artères) et des bâtiments emblématiques, se focalisant sur la valeur " artistique » de l'architecture et de l'urbanisme. On citera parmi les plus importants, le concours portant sur les projets de reconstruction de cinq places moscovites (1931), le concours de l'Académie militaire Frounze (1931), le concours du Palais de la Technique (1933), le concours du Narkomtiajprom [Commissariat à l'industrie lourde] (1934), le concours pour le siège du présidium de l'Académie des sciences (1934-1935), le concours pour le plan d'aménagement de l'Exposition agricole pansoviétique (1935), le concours du Grand cinéma académique (1936), le concours de l'Aérogare (1939), le concours pour le Combinat de la revue Izvestia (1938-1940),

17. Dvorec sovetov, Vsesojuznij konkurs 1932 [Concours pan-soviétique du Palais des soviets de 1932], Moscou : 1933, p. 56. 


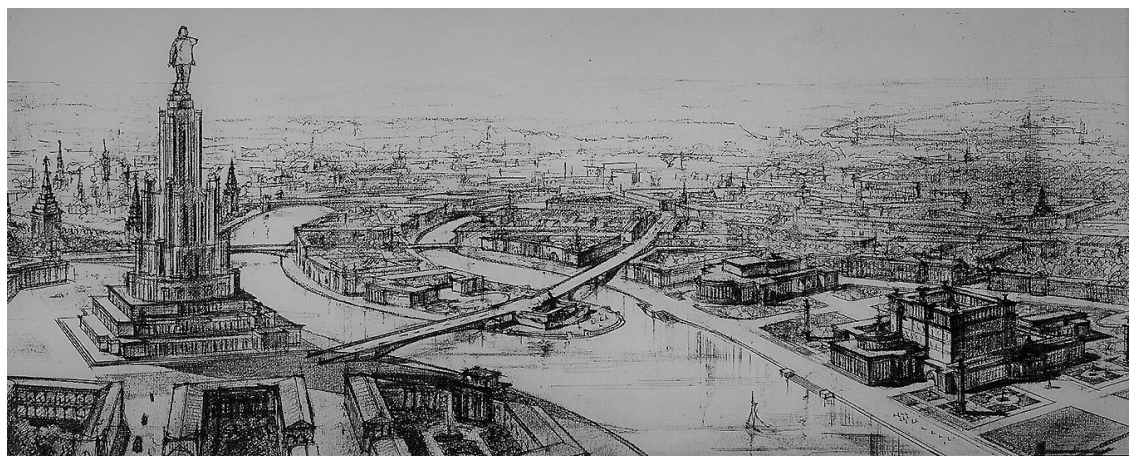

Ill. 3 : A.V. $\hat{S}$ usev (1873-1949), Vue axonométrique du Palais des soviets, du pont Sojmonovskij et du quai de Crimée avec le projet pour le présidium de l'Académie des sciences, 1939. () Collections du Musée d'état d'architecture, A.V. Ŝ usev (Moscou).

enfin le concours pour l'aménagement du quartier de Zaryadie et l'édification du gratte-ciel du Conseil des commissaires du peuple (1940-1941) (ill. 3).

Autre renversement fondamental de cette période, aussi bien technique qu'éthique, les concours ne garantissent plus aux lauréats l'attribution du chantier et la reconnaissance des droits d'auteur. Rappelons que le respect de ces droits, avait été l'un des principaux combats des architectes durant les années 1920. Le concours pour l'hôtel Moskva en 1931, est remporté par les jeunes architectes constructivistes Léonid Savelev (1903-1965) et Oswald Stapran (1901-1984) ${ }^{18}$. Dès 1933, la municipalité de Moscou, attribuera le chantier à l'académicien Aleksej Ŝsev (1873-1949) pour en modifier formellement l'architecture. Qualifié d'abord de co-auteur, Susev sera par la suite désigné comme l'unique auteur du bâtiment ${ }^{19}$. Autre cas intéressant, le concours pour le nouveau thêâtre de Vsevolod Meyerhold à Moscou, fut remporté en 1930 par de jeunes architectes constructivistes, Mihail Barhin (1906-1988) et Sergeï Varhtangov (1907-1987). La construction débute en 1932, la salle est quasiment terminée, mais dès 1933 un nouveau concours est organisé ayant pour objet "l'enrichissement" de la façade constructiviste. Remporté par Aleksej Ŝusev, ce dernier s'attribue rapidement la paternité de la totalité du bâtiment.

L'histoire ne s'arrête pas là. Après 1937, moment de disgrâce pour l'académicien Ŝsev, un autre architecte, Dmitri Čečulin (1901-1981), reprend le chantier et deviendra à son tour, le seul auteur mentionné dans les textes officiels et la littérature spécialisée. Autrement dit le bâtiment, produit de cinq architectes différents, devait rester à la postérité comme l'œuvre d'un architecte, seul légitime puisque étant le dernier choisi par les autorités. Pour l'ingénieur Nikolai Šestopal, qui écrit en 1937 un article incendiaire contre l'académicien Ŝsev, les concours fermés, rendraient le système d'attribution des commandes plus opaque, favorisant leur concen-

18. "Pis'ma v redakciju. Žizn i dejatel'nost' arhitektora Śuseva " [Lettres à la rédaction. La vie et l'œuvre de l'architecte Ŝsev] // Pravda, 1937, nº 239, 30 août, p. 4.

19. RGALI, fond. 2423, op. 1, F. 176, L. 8. 


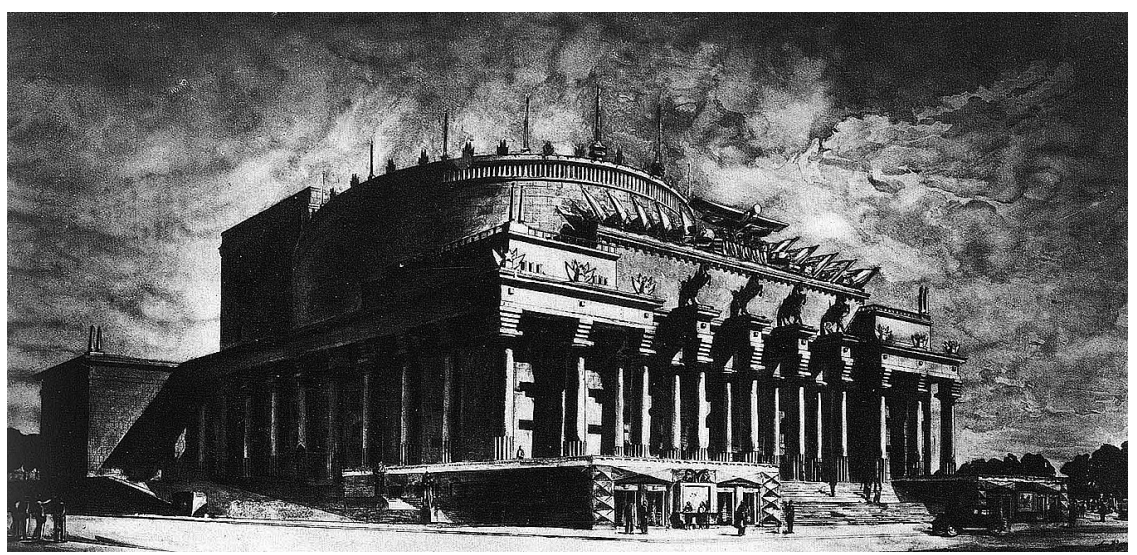

Ill. 4 : Lev Rudnev (1885-1956) et Vladimir Muntz (1903-1974), Projet de concours pour le théâtre de l'Armée rouge, 1932-1933. (C) Collections du Musée d’état d’architecture, A.V. Ŝs usev (Moscou).

tration dans les ateliers des architectes occupant une position élevée dans le système professionnel, au sein de l'Union des architectes ou de l'Académie, et bénéficiant d'un solide réseau dans l'appareil du Parti ou dans les organes de sécurité ${ }^{20}$. Le concours pour le théâtre de l'Armée rouge en 1932-1933, une compétition pourtant fermée entre trois ateliers présélectionnés, ne donnera lieu à aucune recommandation pour exécution, et dès 1934 le chantier sera confié à Karo Alabian, le puissant président de l'Union des architectes (ill. 4).

Ces cas divers montrent la dévaluation complète du concours en tant qu'institution et mécanisme transparent de décision publique, et les dérives du système hiérarchique stalinien. Après l'interruption provoquée par la seconde guerre mondiale, les programmes de reconstruction d'après-guerre redonnent à la pratique des concours un vaste champ d'application. La création en 1943 du Comité des affaires architecturales du Conseil des commissaires du peuple, centralise l'ensemble de l'activité de conception architecturale et urbanistique et notamment l'organisation des concours pour les plans de reconstruction des villes soviétiques. Ce sont pour la plupart des concours fermés, commandant des projets à des architectes renommés de Moscou ou de Leningrad qui siègent souvent eux-mêmes dans les commissions du Comité des affaires architecturales. Le Comité confiera au puissant académicien Aleksej Ŝusev, en 1944, la reconstruction de la ville détruite de Novgorod, et en 1945, la reconstruction de la ville de Kichinev. En octobre 1944, le concours pour la reconstruction de Stalingrad conduira au choix du projet de Karo Alabian (1897-1959), président de l'Union des architectes. Les concours les plus médiatisés portent sur la reconstruction monumentale de centres villes, à Kiev, Minsk, Stalingrad, Riga, Vilnius, Tallinn, Alma-Ata, mais on note également un nombre infini de concours

20. "Pis'ma v redakciju. Žizn i dejatel'nost' arhitektora Ŝuseva » [Lettres à la rédaction. La vie et l'œuvre de l'architecte $\hat{S}$ usev] // Pravda, 1937, n 239, 30 août, p. 4. 


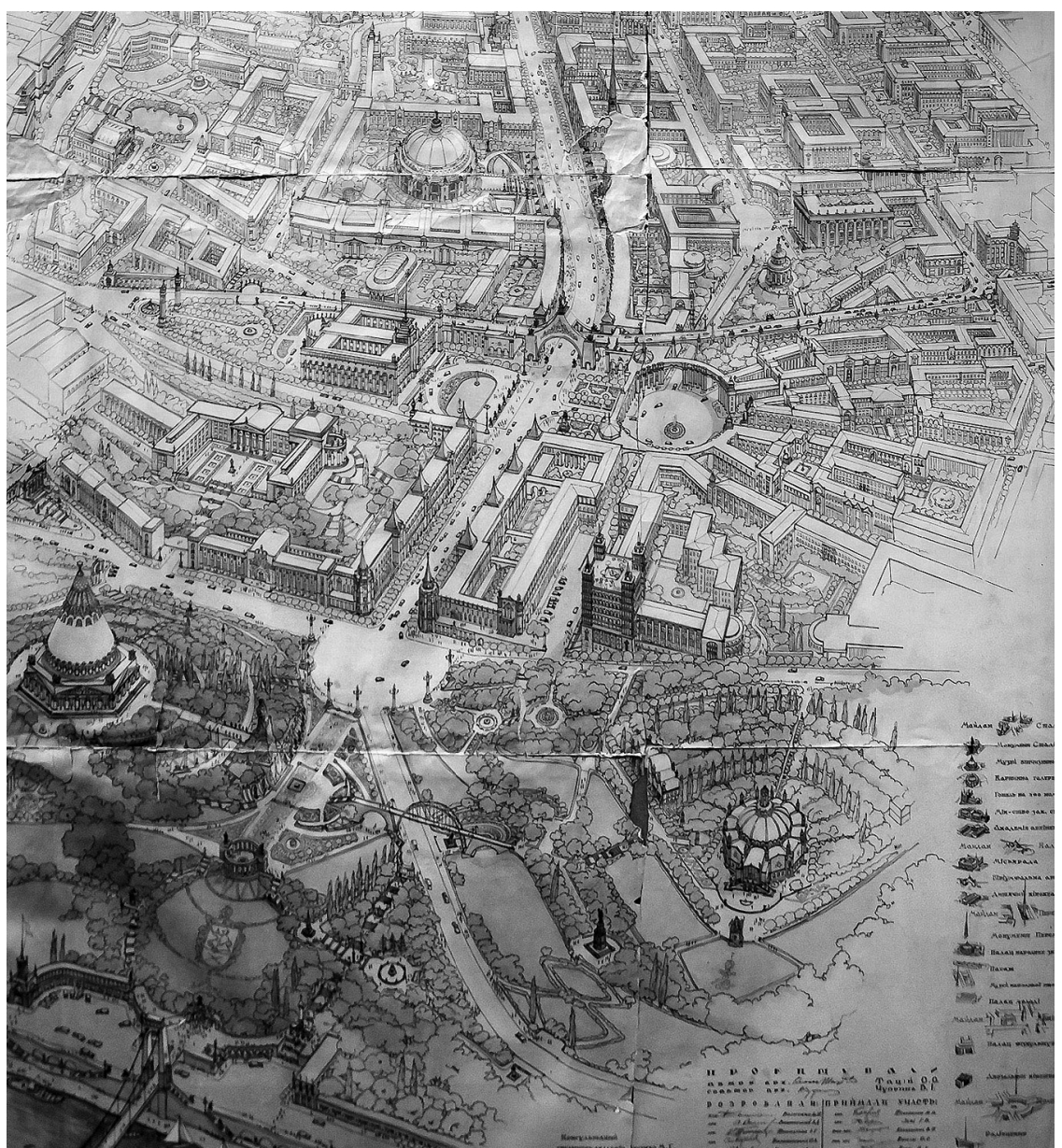

Ill. 5 : Atelier de l'architecte ukrainien Oleksij Tacij (1903-1967), Projet de concours pour la reconstruction du Krešs atik à Kiev, deuxième tour fermé, 1946. (C) Archives de la Bibliothèque scientifique d'état sur l'architecture et la construction, V.I. Zabolotnij, Kiev.

portant sur des sujets très divers qui peuvent recouvrir la totalité de la chaîne de construction, depuis des procédés techniques jusqu'aux arts appliqués (ill. 5). On relèvera ainsi en juin 1945, un concours pan-soviétique pour la création de nouveaux motifs pour des papiers peints avec frises, produits industriellement ${ }^{21}$.

En 1946, une nouvelle réglementation juridique s'applique aux concours avec pour corollaire l'institution d'une nouvelle conception du droit d'auteur : l'auteur du bâtiment, est celui qui l'a construit, et non plus celui auquel appartenait l'idée et la conception du projet. Cette nouvelle législation, est en fait la négation de la 
reconnaissance du droit d'auteur et de la valorisation du travail créateur, qui étaient parmi les fondements essentiels des concours. L'interprétation stalinienne du concours d'architecture trouvait ici son aboutissement, en créant une nouvelle sorte d'architecte "visualisateur " et non visionnaire, réduit à compiler les besoins et les tendances générales de l'État ${ }^{22}$.

Stéphane GAESSLER Doctorant en Histoire de l'art à l'université Paris IV-Sorbonne, Chargé d'études et de recherches à l'INHA

22. Tatjana Prudnikova, "Arhitekturnij Konkurs v Kontekste Kumuliativizma "// Izvestia RGPU A.I. Herzen, Saint-Pétersbourg, 2014, p. 125. 
